\title{
RE-INFECTIONS OF PIG FARMS WITH EP AND APP IN RESPIRATORY-DISEASE- FREE REGIONS OF SWITZERLAND - A SEARCH FOR CAUSES
}

\author{
Hege R. ${ }^{1,2}$, Zimmermann $W^{2}$, Stärk K.D.C. ${ }^{1}$ \\ ${ }^{1}$ Swiss Federal Veterinary Office, Berne. ${ }^{2}$ Clinic for Food Animals and Horses, University of Berne
}

The Swiss Veterinary Services are pursuing the objective to eradicate enzootic pneumonia (EP) and Actinobacillus pleuropneumoniae (APP) in the entire area of the country using partial and full depopulation strategies. Since the first area-wide sanitation in a pilot area was completed in 1996, the problem of re-infection occurred regularly. The main reason appeared to be the purchase of subclinically infected animals. However, a number of re-infections remained unexplained and a search for possible risk factors was initiated using a case-control study approach.

The objective of the study was to describe and identify risk factors for re-infection in areas with a past sanitation. The analysis was based on the comparison of pig farms that were re-infected in the year 2000 and located in areas sanitised in the years 1996 until 1999 and pig farms that were exempted from re-infection in the same area, i.e. in the same period after sanitation. All farms participating in the study were located in the cantons of Berne, Lucerne and Solothurn. All re-infections of both breeding and fattening farms were registered by the Swiss Pig Health Service. All re-infected farms were elected as cases for the study. A random sample of controls was selected out of the remaining farms in the specified areas. Detailed data were collected using farm visits and a structured interview based on a questionnaire. Information on risk factors related to location, management and animal trade was recorded. A second questionnaire was sent to 212 dealers and transport companies covering topics such as structure, organisation and conduct of transports. These data will be used to investigate a potential association between procedures applied by particular dealers and the number of re-infections in farms serviced by them.

Preliminary results showed that in the year 2000, out of a total of 3,983 farms, 107 farms were re-infected (103 cases of EP and 4 cases of APP) in the selected areas of the three cantons. This resulted in an incidence of $0.1 \%$ for APP and $2.6 \%$ for EP. Breeding farms had an incidence of $1.7 \%$, mixed breeding-fattening farms $2.4 \%$ and fattening farms $3.4 \%$.

The cause of re-infection was identified in $69.2 \%$ of all cases. The main reasons were: purchase of animals (43.0\%), air-borne infection (22.4\%), chronicle infection (3.7\%). During the study, 214 farms were visited (107 cases, 107 controls). Preliminary results on the risk factor analysis will be presented at the conference. 